\title{
Analysis of Wireless Mesh Structures Using Localized Backbone Constructions
}

\author{
S. Smys and Jennifer S. Raj
}

\begin{abstract}
In wireless domain finding the dominating set is one of the solutions for connectivity maintenance. Due to the dynamic topology, i.e. nodes move anywhere at any time: finding the dominating set also is a major task and lots of control messages require updating every change that occurs. To overcome the existing issues a new localized mechanism is proposed in this paper named as Localized Backbone Structure (LBS), which uses self stability nodes to alleviate the fundamental wireless problems like connectivity and coverage. And also the proposed work is involved in the research challenged topic topological control. Localized Virtual Backbone nodes have the ability to reduce the link and nodes for active information exchange based on local computations. The work analyses the QoS parameters like scalability with different backbone size and packet delivery ratio in network simulator.
\end{abstract}

Index Terms-Distributed approach, connectivity maintenance, localized protocol, quality of service.

\section{INTRODUCTION}

Wireless network mainly suffer from blind flooding/ broadcasting (i.e., when a source sends a message, all its neighbors will hear it). Due to the broadcast nature, excessive redundant transmissions are generated and may cause a serious problem, referred to as the broadcast storm problem [1]. To alleviate this problem virtual backbone routing is proposed, where set of nodes act as a backbone which makes the routing decision. From the graph theory, a set in graph $\mathrm{G}(\mathrm{V}, \mathrm{E})$ is dominating if all the nodes in the system are either in the set or neighbors of nodes in the set. A connected dominating set (CDS) [2], [3] can form an interesting virtual backbone. Existing research papers [4]-[7] focus on the backbone formation with minimum dominating nodes (named as MCDS - Minimum Connected Dominating Set) and low scalable connectivity maintenance mechanisms. The proposed work involves in the process of topology control with minimum virtual nodes.

Two set of related works are performed; firstly virtual structure based works are identified and the topology control applications are analyzed in the second level. D. Kim et al. propose two centralized backbone formation algorithm in [2], having constant performance ratios for its size and diameter of the constructed CDS, the size of CDS computed by the second algorithm (CDS-BD-C2) is no more than 6.906 times of its optimal solution. It mainly focus on two important metrics of network, such as diameter and average

Manuscript received January 4, 2014; revised April 30, 2014.

S. Smys is with the Department of Information Technology, Karpagam College of Engineering, Coimbatore, India (e-mail: smys375@gmail.com).

Jennifer S. Raj is with the Department of ECE, Karunya University, Coimbatore, India (e-mail: jennifer.raj@gmail.com). hop distances. H. Yang et al. in [3] developed a distributed algorithm for k-hop dominating set, which uses topology update packets or route request packets for reducing flooding search space in the dominating set, resulting in significant flooding overhead reduction in broadcast related applications. The approach can be verified in a distributed manner by a node that has only the link information of its neighbors. T. Qiang et al. in [4], proposed a virtual backbone tree based energy efficient protocol (VBTEBHRP), which is used to improve the energy efficiency and the network life time. In [5]-[9], the authors proposed various localized algorithms for backbone construction and concentrating on different parameters like high mobility, energy consumption and backbone size in ad hoc domain.

F. Wang et al. in [10], proposed algorithm named as connected dominating set augmentation(CDSA), which is used to construct a two connected dominating set which can resist the failure of one wireless node. Localized structure must have Self organization ability, this property is studied from [11]-[13]. An energy efficient connected dominating set is given in [14], which do not involve in the process of connectivity analysis. The remainder of the paper is organized as follows: section II presents the definition of the proposed work. Section III describes the construction virtual structure with mathematical proof. Section IV shows the simulation results and performance analysis. Finally section $\mathrm{V}$, concludes the paper.

\section{DEFINITION AND NotATION}

Localized Virtual Structure (LBS): It is constructed based on node degree. If a node wishes to communicate LVS, it must be present in its transmission range. LVS node has the ability to handle at least one neighbor. In the simulation node degree 1, 3 and 5 are also analyzed with virtual structure. It is also named as Connected dominating set (CDS) or virtual node. (In the simulation LVS-ND5, 3 and 1 named as localized virtual structure

Leaf Node (LN): The Leaf Node is defined as a normal node not involve in control transfer.

\section{LOCALIZED VIRTUAL STRUCTURE FORMATION}

Initially the network is modeled using unit disk graph with $\mathrm{V}$-vertices and E-edges, say $\mathrm{G}(\mathrm{V}, \mathrm{E})$. The proposed work follows node degree as a key parameter to construct the virtual structure. For alternate routing, minimum degree backbones are used in one hop i.e. a backbone node is able to handle maximum five connections and minimum of one connection. The same is also applicable during the movement of node. A simple marking scheme is used to 
construct the virtual structure. If a node becomes a LVS node it must have high degree (in this paper node degree with at least 1,3 , and 5 is considered), otherwise it becomes a normal node (a leaf node in the tree). The topology control (connectivity) and maintenance is supported by LVS based on the node addition or deletion of inactive nodes in the network. The entire construction process is given in Fig. 1 .

\section{A. Algorithm for Localized Virtual Structure Formation}

The localized structure with connectivity maintenance is based on marking scheme with tree. LVS nodes are formed based on the following marking scheme "A node is marked true if it has three unconnected neighbors or with node degree higher than three and each neighbor is connected with one leaf node. A set of marked nodes form the localized virtual structure". Virtual structure formation based on hierarchical architecture as follows: 1. LVS node with high degree 2.virtual node with degree three and 3.leaf node (attached with virtual structure). The hierarchical order provides many connections in one hop and performs the alternate routing during movement. After the virtual structure construction, each node involve in the routing, information processing and maintain the sleep node information for topology control. To maintain the topology and connectivity, leaf nodes are resumed by the virtual structure when it is required. This process mainly supports the low energy consumption of each node and also used to improve the network life time. Finally normal node (also named as leaf node) involves in information exchange process only and it will become backbone node at the time existing backbone fails. In the proposed work node degree is counted only from the virtual node to leaf nodes, not between the virtual nodes.

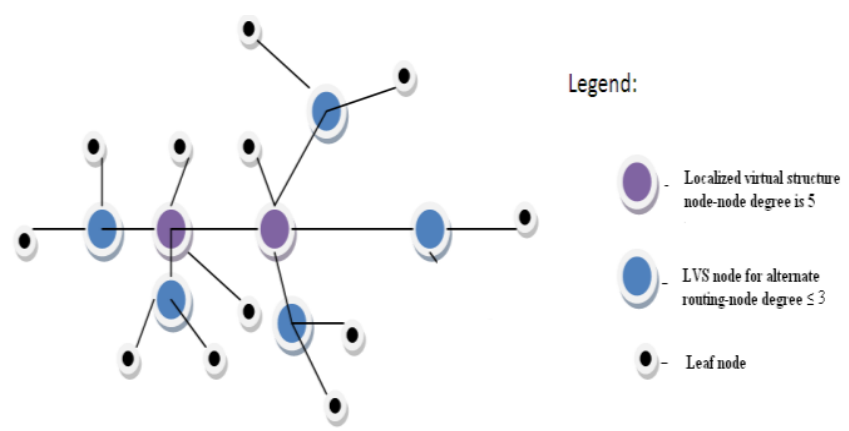

Fig. 1. Virtual structure construction based on node degree.

\section{Algorithm 1 for Virtual Structure Formation:}

(In the proposed, work node degree is counted only from the virtual node to leaf nodes, not between the virtual nodes).

1: INPUT: An undirected disk graph $G=(V, E)$

2: OUTPUT: A connected localized virtual structure $L$

3: Construct Tree $\mathrm{T} \leftarrow\{V, E\} / /$ with at least one unconnected neighbors

4: Define a localized virtual set from T. // initially node degree 3 or 5 based virtual structure is constructed, if it is not exist construction follows at least one degree (one degree $=$ leaf node + virtual node)

5: $L(v) \in\{G\}$, where $L \geq 1,3$ or 5

6: Find a subset A for alternate routing from $L$, such that $\left(l_{1}, l_{2} \ldots . \ln \right) \in \mathrm{V}$

7: if $l 1, l 2$ alternate route virtual nodes then

$/ /$ node degree $l_{1}, l_{2} \geq 1$ or 3
8: Connect leaf node $\mathrm{n}$ to $L$ or $l$

9: Construct Tree $T \leftarrow L U l U n(v)$

10: while $L(v)$ change the position do // $v, u$ - vertices in L(localized structure)

11: $l(u) \leftarrow L(v) . / /$ connection of ' $L$ ' is maintained via ' $l$ '. // $l$ subset of $L$

12: Add $u, v$ only if $l$ connects $v$ and $u$ in one hop

13: similarly other nodes of $L$ added with one hop $l_{1}, l_{2} \ldots$. ln

14: $(l, n) \leftarrow$ update $i / / i$ - state information

15: if $l_{1}$ not available in one hop or not in the transmission range // this is only applicable for low transmission range

16: $n \leftarrow l / /$ leaf node $(n)$ become localized virtual node

\section{B. Correctness}

Now we prove that our algorithm guarantees a node degree based one connected localized virtual structure. We argue that in algorithm: i) in line 4 , virtual structure $(L)$ follows 1,3 or 5 degree and i) in line 11, during the movement connection of $L$ is maintained via $l$. ( $L$-virtual structure, $l_{1}$ alternate route virtual nodes) and iii) in line 16 , $\mathrm{n} \leftarrow l$, leaf node become localized virtual node.

For i), virtual structure formation based on marking scheme, it has been proved in [2] that if a graph $G$ is connected, BFS tree is build first, then find an MIS based on the BFS tree and connect the MIS nodes to form a CDS. For ii) connection of $L\left(v_{1}\right)$ is maintained via $l(u)$ means there always a possible node degree between $L$ and $l$ within the localized virtual set. This is true because $G$ is a localized Tree (T), with sufficient node degree. If one node degree fails or removed from graph $(\mathrm{G})$, virtual structure is maintained with one hop leaf node. For iii) leaf node become localized virtual node, it means that a graph $G$ is connected, the number of CDS is reduced due to high node degree by adding new nodes to leaf node. This is used to maintain the algorithm complexity in mobile condition also and secondly it based on the marking scheme "A node is marked true if it has three unconnected neighbors or with node degree higher than three and each neighbor is connected with one leaf node. A set of marked nodes form the localized virtual structure", it proves final correctness (iii).

\section{Complexity}

Time complexity of LVS in the order of ' $n$ ' and ' $\Delta$ ', where $\mathrm{n}$ is the number of nodes in graph $\mathrm{G}$ and $\Delta$ - node degree. Initially complexity of CDS construction in the order of $\mathrm{O}(n)$ time and after the addition of each node based on node degree $(\Delta)$ the complexity is increased in the order of $\mathrm{O}(n+\Delta)$. It is true if $\mathrm{G}$ follows 1 node degree otherwise complexity is $\mathrm{O}(n+3 \Delta)$ or $\mathrm{O}(n+5 \Delta)$. Hence the complexity of the proposed work is $\mathrm{O}\left(n^{2}\right)$. Secondly the message complexity includes the node degree and intermediate nodes information, hence it should be in the order of $\mathrm{O}(n \Delta)$, where $n=1,3$ or 5 .

\section{Approximation Ratio}

In this section we show that LVS has a constant approximation ratio of 73 .

Let $\mathrm{V}$ be the set of virtual nodes selected to interconnect L (leaf) nodes in LVS. And let 3ND (node degree) and 5ND be an MCDS for the given set of nodes. Then $\mathrm{V}$ and $\mathrm{L}$ is connected and $\mathrm{MCDS}=5 \mathrm{ND}+5|\mathrm{~V} 2|-1 ; \quad \mathrm{CDS}=3 \mathrm{ND}+3|\mathrm{~V} 1|-$ 
1(dominating set includes node degree, leaf node and neglect the forwarding link, V1, 2-virtual nodes with 3 and 5 degree. For each virtual node at least one node degree must be exist, hence $1 \mathrm{ND}+1 \mathrm{~V} 2=2$

Form [6],

$$
\begin{gathered}
|1 \mathrm{CDS}| \leq|8 \mathrm{MCDS}|+1 \leq 8(5 \mathrm{ND}+5|\mathrm{~V} 2|-1) \mid+1 \leq \\
40 \mathrm{ND}+40|\mathrm{~V} 2|-8) \mid+1 \leq 40(\mathrm{ND}+|\mathrm{V} 2|)-8) \mid+1 \\
\leq 40(2)-8|+1,| 1 \mathrm{CDS} \mid \leq 73
\end{gathered}
$$

\section{RESUlt ANALYSIS}

Two sets of analysis are performed in the simulation: Virtual structure with Quality of Service and Topology (connectivity) issues. The self organized backbone is constructed using the marking scheme with tree and sleeping node is periodically turned off and on for topology control and maintenance process. For this purpose the tcl command in ns-2 is modified in $\sim$ ns/indeputils/propagation/threshold.cc. During the off time each leaf node assigned with 0.1 watt and set 0.2814 watt for virtual nodes. All the simulations are conducted using ns-2 with 200 nodes with a $1250 \times 1250 \mathrm{~m}^{2}$ deployment area, $0.90 \mathrm{~s}$ hello interval and random way point model. The following parameters are observed: number of backbone and network size for different node degrees, control message transmitted per nodes and delay of each node. Duration of the simulation run is $300 \mathrm{~s}$ and 256 byte packet size used. During the construction of virtual structure, an effective marking scheme is applied, initially all the nodes are in black color and first level iteration the node degree 5 virtual nodes are colored by red and the node movements are represent by the yellow color.

\section{A. Performance Metrics}

- Scalability: As the number of nodes in wireless network increases, scalability imposes difficulties in transferring data. To overcome this issue, localized algorithm is proposed and initially a localized set of 50 nodes are tested from 200 nodes. The following CDS performance are analyzed in the simulation

- Network size vs. CDS: Number nodes versus connected dominating set as a function of 200 nodes are considered. (1CDS-one hop neighbor information;2CDS-2hop neighbor information)

- Transmission range vs. CDS: Transmission range varies from $100 \mathrm{~m}$ to $300 \mathrm{~m}$ and different localized structures are plotted.

- Node degree vs. CDS: initially network was formed with 3 or 5 degree virtual nodes, node degree will be increased in the order of seven per each node a total of 35 will be added to virtual structure and the percentage of virtual structure requirements are plotted in the result analysis.

- Packet delivery ratio: It is calculated as the ratio between the number of data packets that are sent by the source and the number of data packets that are received by the intermediate or destination node.

\section{B. Simulation Results}

In simulation three types of LVS are analyzed namely
LVS-ND5, 3, 1-Localized Virtual Structure with Node Degree 5, 3, and 1 are used. The comparison mainly focus on node degree as a key factor, since network is formed with a marking scheme "A node is marked true if it has three unconnected neighbors or with high node degree than three, transmission power and each neighbor is connected one leaf node. A set of marked nodes form the localized virtual structure". The number of virtual nodes required per network size is given in Fig. 2. From the comparison node degree with 5 requires nearly $15 \%$ of nodes must act as a virtual node, where as node degree 3 and 1 requires more than $50 \%$ backbone nodes. Due to high node density ND5 is preferred for control exchange and it also need more control load for initial construction and maintenance. The transmission range analysis is based on number of CDS requirement with the different range transmission. Here the range will be increased from 50 to $300 \mathrm{~m}$ and the sizes of virtual nodes are measured. For $300 \mathrm{~m}$ range only 8 virtual nodes each with 23 degree is required for ND5 (initially degree is 5) to connect 200 nodes.

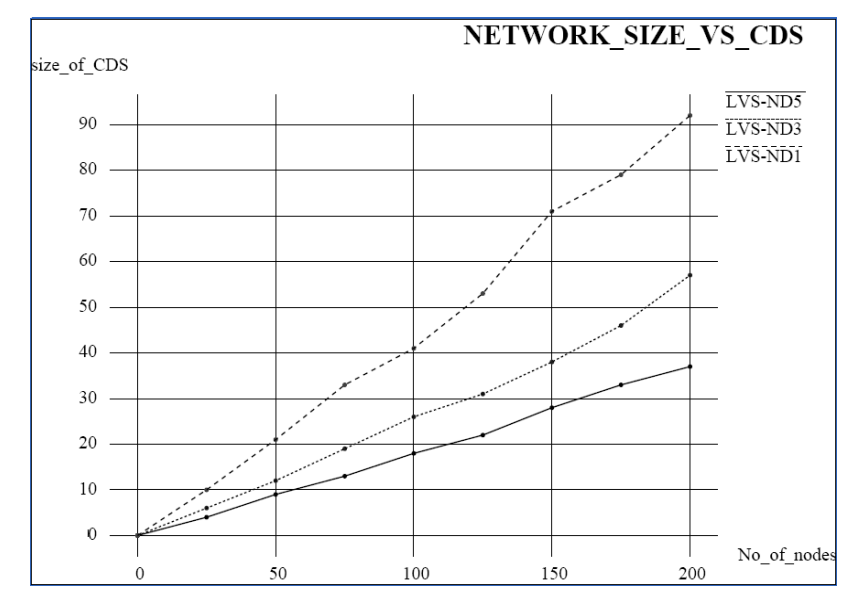

Fig. 2. Network size vs. CDS for various node degree (initial node degree 1, $3,5)$

Other two methods requires 88-18 (ND3) and 51-14 (ND1) virtual nodes for the transmission range of 50-300m. Transmission range and the virtual node requirements are given in Fig. 3. The process follows one connected dominating set i.e. each virtual node construction follows one hop. Similarly two connected dominating set analysis is more complicated than $1 \mathrm{CDS}$, due to 2 hop neighbor information between the backbones. It requires lot of control message for route maintenance and data transfer. The number of virtual nodes is reduced to $50 \%$ than one CDS approach for the same node degree. This process is shown in Fig. 4. Fig. 5 shows the virtual node requirement for different node degree. In this average degree simulation, we show the scalability of LVS approach for a certain network density. Initially network is formed with maximum of 5 node degree after the first round of transmission; node degree is randomly increased for each node.

For each simulation run, we experimented on different speed levels $(5 \mathrm{~m} / \mathrm{s}$ to $30 \mathrm{~m} / \mathrm{s})$ by analyzing the network behavior for $300 \mathrm{sec}$ for LVS. For mobile condition the CDS size is increased gradually to reach the peak value for 33 for node degree 7 and need the minimum of 13 nodes for maximum 35 degree. The stable condition requires only $22-$ 7 for different node degrees. 


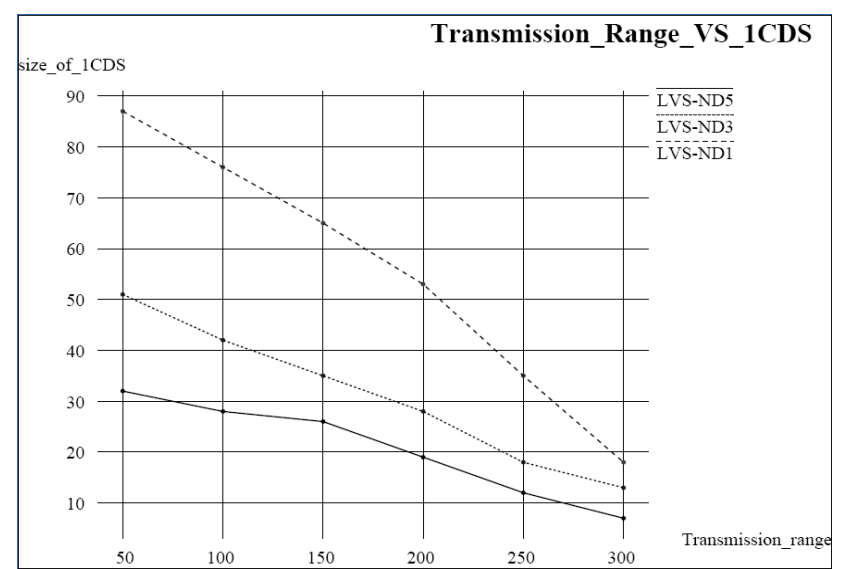

Fig. 3. Transmission range vs. CDS size (1-connected).

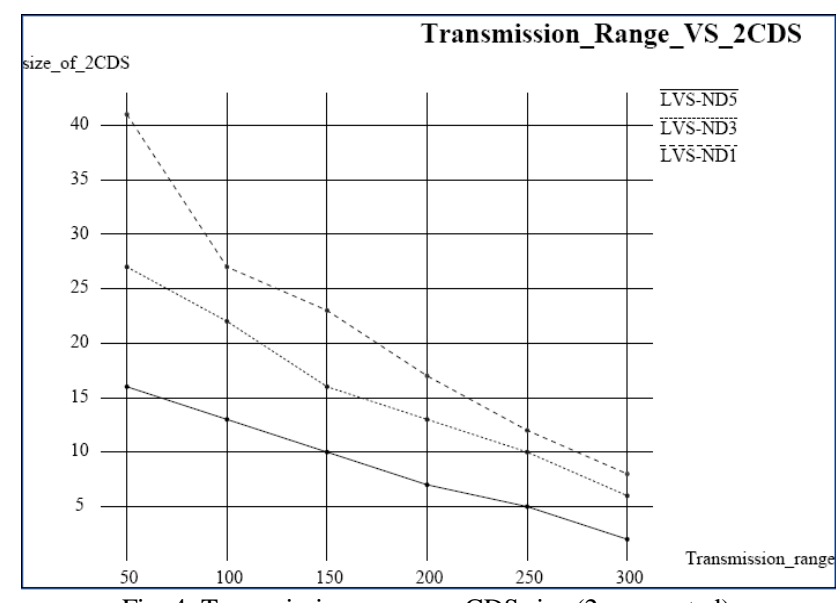

Fig. 4. Transmission range vs. CDS size (2-connected).

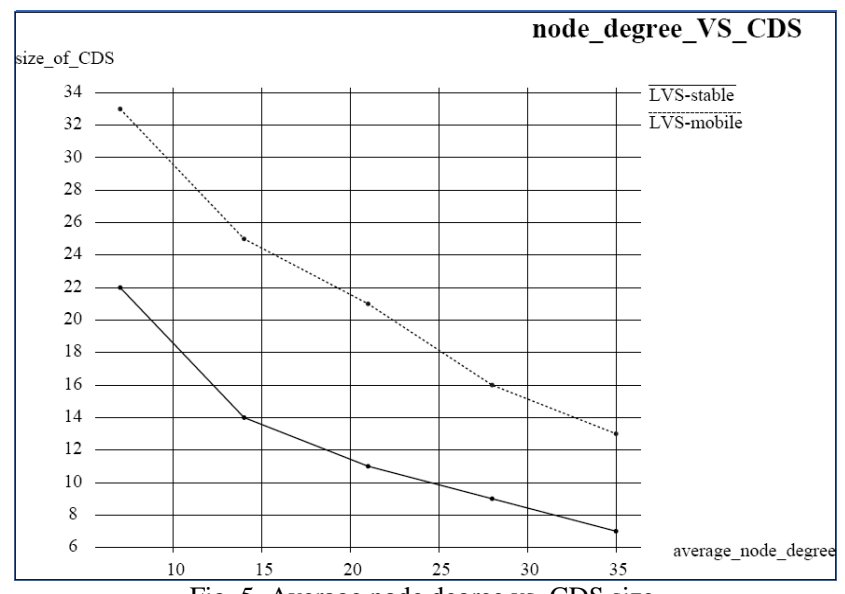

Fig. 5. Average node degree vs. CDS size

\section{CONCLUSION}

The main contribution of this paper is an in-depth analysis of the virtual structure formation with node degree analyses. A large number of algorithms exist to construct a backbone, only very few motivate the concept of 2 connected dominating set and how to perform the connectivity issues with node degree. LVS uses the localized updates in distributed manner with different transmission range and node degree. The advantage of node degree based localized solution gives a clear picture about backbone construction, topology control, and maintenance. The main features of the topology control mechanism based on backbone updated messages, which is send only to nearby node when more connections surround an originating backbone. Topology control mechanism is a very challenging issue in fast moving distributed dynamic networks and this is also included in our paper with virtual structure.

\section{REFERENCES}

[1] Y. C. Tseng et al., "The broadcast storm problem in a mobile ad hoc network," Wireless Networks, vol. 8, no. 2-3, pp. 153-167, 2002.

[2] D. Kim et al., "Constructing minimum connected dominating sets with bounded diameters in wireless networks," IEEE Transactions on Parallel And Distributed Systems, vol. 20, no. 2, pp. 147-157, 2009.

[3] H. Y. Yang et al., "Distributed algorithm for efficient construction and maintenance of connected K-Hop dominating sets in mobile AdHoc networks," IEEE Tran. on Mobile Computing, vol. 7, no. 4, pp. 444-456, 2008.

[4] Q. Tang, B. W. Wang, and Z. C. Dai, "Virtual backbone tree based energy balanced hierarchical routing protocol," in Proc. 2nd International Conference on Power Electronics and Intelligent Transportation System, 2009, pp. 204-208.

[5] S. R. Jennifer and R. Harikumar, "A dynamic overlay approach for mobility maintenance in personal communication networks," International Journal of Peer to Peer Networking with Application, 2012.

[6] M. Min, F. Wang, D. Z. Du, and P. M. Pardalos, "A reliable virtual backbone scheme in mobile Ad-Hoc networks," in Proc. 1st Int. Conference on Mobile Ad hoc and Sensor Systems, 2004, pp. 60-69.

[7] S. J. Lee et al., "Efficient and resilient backbones for multihop wireless networks," IEEE Transactions on Mobile Computing, vol. 7, no. 11, pp. 1349-1362, 2008.

[8] J. S. Raj and R. Harikumar, "A localized computing approach for connectivity improvement analysis in wireless personal networks," Springer-International Journal of Wireless Personal Communications, vol. 72, no. 4, pp. 2867-2883, 2013.

[9] S. Smys and G. J. Bala, "Performance analysis of virtual clusters in personal communication networks," International Journal of Cluster Computing, vol. 15, pp. 211-222, August 2012.

[10] F. Wang, T. Thai, and D. Du, "On construction of 2-connected virtual backbone in wireless networks," IEEE Transaction on Wireless Communication, vol. 8, no. 3, pp. 1230-1239, March 2009.

[11] S. Smys and G. J. Bala, "Efficient self-organized backbone formation in mobile Ad Hoc networks (MANETs)," International Journal of Computers and Electrical Engineering, vol. 38, pp. 522-532, 2012.

[12] S. Smys and G. J. Bala, "Self organized, topology control ability backbone node in wireless networks," International Journal of Wireless Personal Communication, vol. 63, no. 3, pp. 529-548, 2012.

[13] S. Smys, G. J. Bala, and J. S. Raj, "Self-organizing hierarchical structure for wireless networks," in Proc. International Conference on Advances in Computer Engineering, 2010, pp. 268-270.

[14] R. Harikumar and J. S. Raj. Ad hoc node connectivity improvement analysis-Why not through mesh clients? Computers \& Electrical Engineering. (2013). [Online]. Available: http://dx.doi.org/10.1016/j.compeleceng.2013.11.020

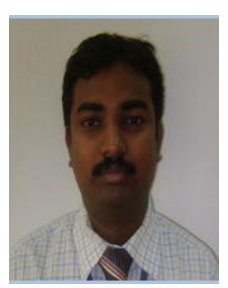

S. Smys received the master's degree in digital communication and network engineering from ANNA University, India and completed doctorate work in wireless networks from Karunya University, India. Currently he is a professor in Karpagam College of Engineering, Coimbatore, India. He is a member of IEEE and IAEng. His research interests are self organization and topology control in mobile networks. $\mathrm{He}$ is also a reviewer for various Springer journals, IET Communication, Elsevier publications and various IEEE conferences and publishes more than thirty research articles in Journals and International conferences.

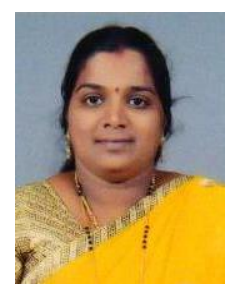

Jennifer S. Raj received the master's degree in communication system from SRM University, India. Currently she is pursuing Ph.D. in information and communication engineering at Anna University of Technology, Coimbatore, India. Her interests are in wireless networks with self organization and topology control structures. She is a life member of ISTE, India. She is book reviewer for Tata Mc Graw hill publication and publishes more than fifteen research articles in the journals and IEEE conferences. 